\title{
DES ARTISTES ENGAGÉS AU BURKINA FASO
}

Rappeurs burkinabé, trajectoires artistiques et contournements identitaires

Anna Cuomo

\section{De Boeck Supérieur | «Afrique contemporaine »}

2015/2 n $254 \mid$ pages 89 à 103

ISSN 0002-0478

ISBN 9782807300743

Article disponible en ligne à l'adresse :

http://www.cairn.info/revue-afrique-contemporaine-2015-2-page-89.htm

\section{!Pour citer cet article :}

Anna Cuomo, « Des artistes engagés au Burkina Faso. Rappeurs burkinabé, trajectoires artistiques et contournements identitaires », Afrique contemporaine 2015/2 (n² 254), p. 89-103.

Distribution électronique Cairn.info pour De Boeck Supérieur.

(C) De Boeck Supérieur. Tous droits réservés pour tous pays.

La reproduction ou représentation de cet article, notamment par photocopie, n'est autorisée que dans les limites des conditions générales d'utilisation du site ou, le cas échéant, des conditions générales de la licence souscrite par votre établissement. Toute autre reproduction ou représentation, en tout ou partie, sous quelque forme et de quelque manière que ce soit, est interdite sauf accord préalable et écrit de l'éditeur, en dehors des cas prévus par la législation en vigueur en France. Il est précisé que son stockage dans une base de données est également interdit. 


\title{
Des artistes engagés au Burkina Faso Rappeurs burkinabé, trajectoires artistiques et contournements identitaires
}

\author{
Anna Cuomo
}

\begin{abstract}
L'article aborde différentes circulations de rappeurs burkinabé au sein de mondes culturels et militants, locaux comme globaux. II cherche à comprendre de quelle manière la catégorie d'" artiste engagé " peut constituer à la fois une ouverture vers d'autres réseaux, mais aussi une forme d'enfermement conduisant ces artistes à la contourner pour s'en émanciper. Les artistes contemporains étudiés mettent en place différentes stratégies de contournement au sein de leur carrière musicale, laissant entrevoir des appartenances sociales multiples allant d'une certaine élite économique et culturelle à des milieux plus populaires. Ces ruptures correspondent à une volonté de s'extraire d'un milieu dans lequel ils ont été catégorisés, et une ambition d'avancer vers une reconnaissance plus large.
\end{abstract}

Mots clés : Burkina Faso - Rap - Anthropologie - Artistes engagés - Subjectivités

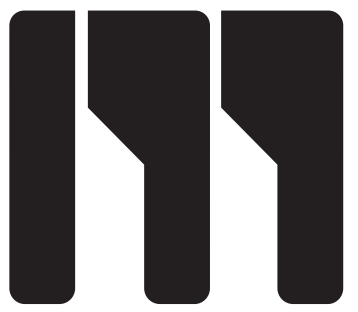

Musique désormais globalisée, le rap arrive au Burkina Faso - plus généralement en Afrique de l'Ouest - dans les années 1980 par le biais d'une jeunesse aisée, qui ramène de ses voyages en Europe et aux États-Unis des cassettes, des vidéos, des vêtements. D’abord écouté dans un milieu restreint, il se popularise dans les années 1990 avec le développement des médias et surtout des radios libres (Frère, 2004). Puis des rappeurs burkinabé émergent, reconnus par leur public et les acteurs culturels comme des «artistes engagés ${ }^{1}$ », s'inscrivant dans un contexte politique qualifié de « régime semi-autoritaire » (Hilgers, Mazzocchetti, 2010).

En parallèle, l'industrie émergente du rap en Afrique francophone s'organise peu à peu en réseaux, à travers la création de festivals dédiés à la

Anna Cuomo est doctorante au département d'anthropologie de l'EHESS et doctorante associée à
I'Institut des mondes africains

(IMAF). 
culture hip-hop ${ }^{2}$ dans plusieurs pays (Gabon, Sénégal, Niger, Burkina) où circulent promoteurs musicaux et acteurs culturels. Certains rappeurs burkinabé commencent ainsi à multiplier les expériences scéniques, localement dans des contextes très divers (concerts de quartiers, manifestations militantes, festivals), mais aussi à l'échelle internationale. Cet article étudie ces expériences circulatoires (Le Menestrel, 2012), entendues non pas uniquement comme des circulations du Sud vers le Nord, mais aussi comme des circulations au sein de mondes sociaux différents (Augé, 1994 ; Becker, 1988) dans une même localité. Il est plus particulièrement axé sur la catégorie d'« artiste engagé », qui, localement, peut renvoyer à différents domaines (politique, social, culturel). Comment l'image d'«artiste engagé » acquise par certains rappeurs peut-elle constituer une ouverture vers d'autres mondes que celui du rap ? Dans quels contextes et avec quels enjeux cette « étiquette » peut-elle être vécue comme réductrice, voire contraignante?

À partir d'enquêtes ethnographiques menées depuis 2010 à Ouagadougou et en France dans le cadre d'une thèse d'anthropologie sur les rappeurs burkinabé, l'article se penchera dans une première partie sur l'inscription de Smockey et du groupe Faso Kombat dans les mondes de la danse contemporaine et du théâtre burkinabé. La deuxième partie sera axée sur des expériences de rupture avec cette «étiquette », à travers l'évolution de David, ancien membre de Faso Kombat et de Smarty, ancien membre de Yeleen ${ }^{3}$. Loin de les analyser comme une dépolitisation de ces artistes, cet article propose de les appréhender comme des processus de subjectivation. L'objectif est de « comprendre l'opposition dynamique entre les assignations identitaires et l'émergence d'un sujet décentré qui s'émancipe de ces assignations » (Agier, 2013, p. 191).

\section{Intégrer d'autres mondes grâce à une image d'artiste engagé}

Aujourd'hui, il n'existe pas un monde du rap à Ouagadougou : rappeurs et acteurs culturels liés à cette pratique artistique s'intègrent plutôt à une multitude de mondes de l'art (Beker, 1988). Ainsi, certains rappeurs sont plébiscités par des chorégraphes ou des metteurs en scène privilégiant dans leurs créations la dialectique art/politique et la rencontre entre différentes pratiques artistiques. Ces collaborations constituent une ouverture sur de nouvelles expériences, induisant un travail, des codes et des publics différents et permettent à des rappeurs burkinabé de diffuser leur musique dans d'autres réseaux.

Le rap s'impose parmi les autres genres nationaux dans les années 2000 grâce à des figures émergentes cherchant à légitimer leur place dans la musique

1. Cette catégorie renvoie à un engagement au sens large, elle est reprise et utilisée par de nombreux acteurs qui n'en ont pas la même définition.
2. Née à New York, dans le Bronx, dans les années 1970, la culture hip-hop regroupe plusieurs modes d'expression (danse, rap, graffiti, djing) et s'est développée suite à des violences entre gangs, dans un contexte post-droits civiques. Cette culture, désormais globalisée, rassemble de nombreux codes vestimentaires, esthétiques et idéologiques en constante évolution.

3. "Lumière » en dioula. 
burkinabé. Cette intégration passe par un processus d'appropriation du rap au sein d'un espace musical national dominé par le genre «tradi-moderne ». Nommé ainsi par les acteurs culturels et artistes locaux, il renvoie à toute chanson s’inspirant du «terroir » burkinabé (rythmes, instruments ou chants reconnus comme traditionnels), enregistrée avec des instruments occidentaux

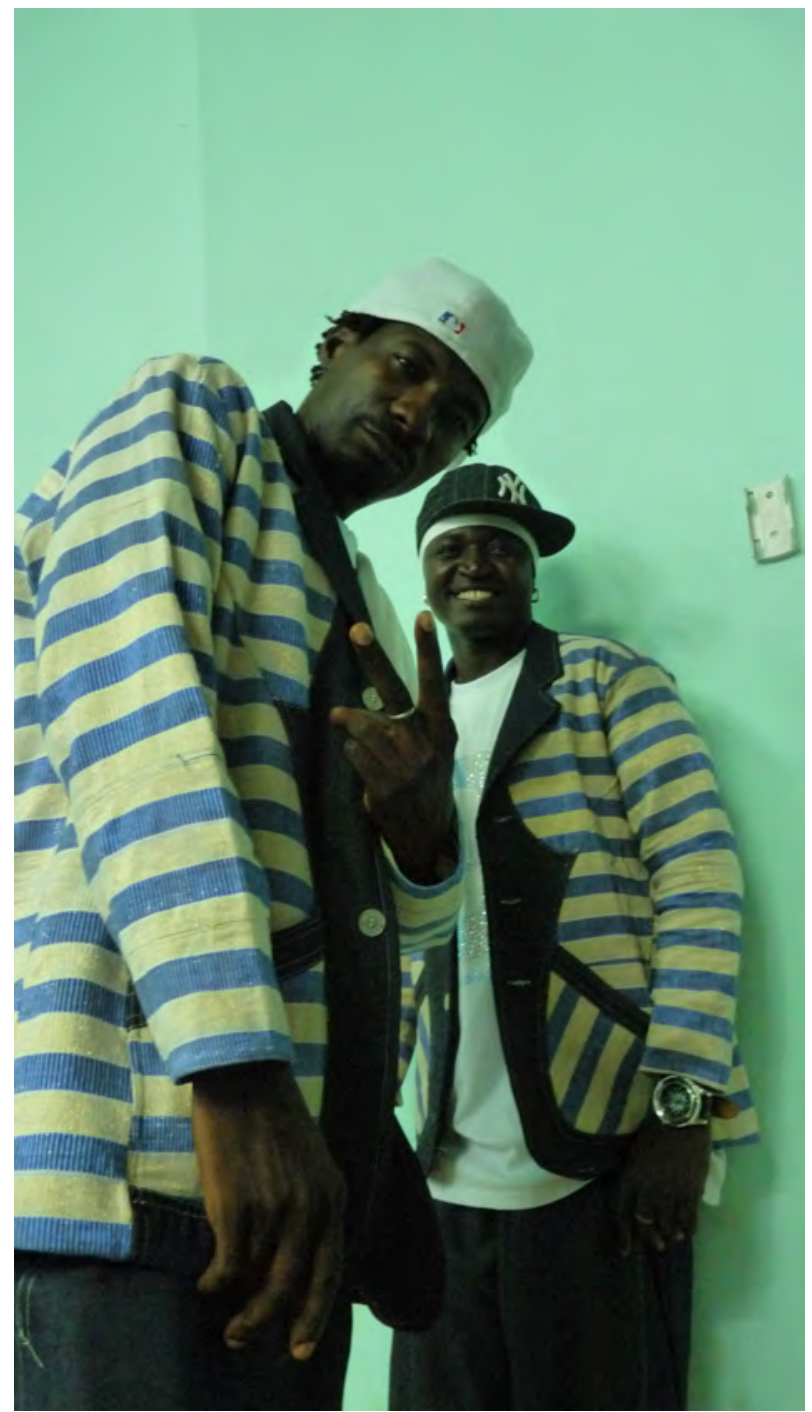

Faso Kombat, entre musique et théâtre. Faso Kombat, comme Smockey et Yeleen, s'imposent sur la scène musicale burkinabé au début des années 2000. Ils poursuivent aujourd'hui leur carrière en solo. Composé de David et Malkhom, le groupe fut engagé dans une pièce de théâtre écrite et mise en scène par Aristide Tarnagda, comédien, écrivain et metteur en scène burkinabé. Et si je les tuais tous, madame? met en scène le monologue d'un exilé, parti à l'aventure en abandonnant ses proches, posté à un feu rouge, qui tente de communiquer avec une automobiliste en lui exprimant ses déceptions, ses angoisses, ses contradictions et sa volonté d'avancer. Le comédien Lamine Diarra est accompagné sur scène par Faso Kombat et un chanteur burkinabé.

Photo de Anna Cuomo, Maison du peuple de Ouagadougou, Burkina Faso, 2 juillet 2011. 
ou avec la MAO (musique assistée par ordinateur). Ce genre est soutenu par les institutions culturelles locales comme internationales, qui y voient une affirmation identitaire nationale permettant à la culture burkinabé de s'exporter dans le contexte de mondialisation (Daboué, 2014). Ce processus permet ainsi à certains d'être reconnus nationalement d'un public élargi : «Au début, on nous prenait pour des voyous et le rap était mal vu. Il fallait qu'on trouve un moyen d'être écoutés et appréciés des Burkinabé. Il a fallu ralentir le flow, traiter de thèmes proches de nos réalités, s'inspirer de chants et rythmes de nos villages », affirme par exemple $\mathrm{Smarty}^{4}$, ancien membre du groupe Yeleen qui a connu un succès important pendant toute la décennie 2000, et qui évolue désormais en solo. Cette stratégie s’inspirait des rappeurs sénégalais ou ivoiriens, déjà passés par cette phase d'appropriation du rap (Martin, 2014). Au-delà d'une reconnaissance locale ${ }^{5}$, la construction d'une coloration africaine sélective et esthétisée permet à ces artistes de s'exporter en proposant des productions musicales originales et africaines, tout en étant « branchées » à des éléments culturels globalisés (Amselle, 2001), issus en partie de l'« Atlantique noir ${ }^{6}$ ».

Devenus de «nouvelles figures de la réussite» (Banégas, Warnier, 2001), Smockey, Faso Kombat et Yeleen s'imposent sur la scène musicale burkinabé au début des années 2000 et sont les seuls issus de la première génération à continuer aujourd'hui. Malgré la reconnaissance acquise par ces rappeurs, le Burkina Faso ne possède pas de véritable industrie musicale : il s'agit plutôt de dynamiques individuelles, carriéristes, cherchant à élargir leur public et multiplier les invitations à se produire sur scène, sans contrat de diffusion ni sponsor.

En parallèle, depuis les années 2000, la capitale, Ouagadougou, est devenue le théâtre d'une pléthore de festivals internationaux ${ }^{7}$ dédiés à la danse (Dialogues de corps, Festival international de danse de Ouagadougou), à la musique (Saga Music, Jazz à Ouaga), au hip-hop (Waga hip-hop), au théâtre et aux arts de rue (Les Récréatrales, rendez-vous chez nous), au cinéma (Festival panafricain de cinéma de Ouagadougou), aux droits humains (Ciné Droit Libre, Festival international de la liberté d'expression et de la presse)... Ces manifestations constituent de véritables carrefours, des lieux de rencontres aux

4. Entretien effectué avec Smarty, Paris, juin 2015.

5. En accord avec Judith Butler (2006, p. 15), je considère la reconnaissance comme "un site de pouvoir pour lequel l'humain est produit de façon différentielle ». En effet, ces artistes ne cherchent pas une reconnaissance égalitaire ou équitable, ils cherchent à obtenir une forme de pouvoir.

6. L'« Atlantique noir » fait référence à un vaste espace géographique lié historiquement par le commerce triangulaire et l'esclavage. Selon Gilroy (2003), il signifie désormais un espace culturel fait de circulations, d'hybridités, de rapports de pouvoir et de modernité. Voir également Cuomo (2014).

7. Ces festivals sont à l'image d'une importante vie associative au Burkina Faso depuis les années 2000, particulièrement à Ouagadougou, et sont soutenus par une politique culturelle cherchant à valoriser ce genre d'initiatives.

8. En 2006, il fit appel au groupe Faso Kombat pour la création «a bengue ", néologisme évoquant les jeunes Africains qui passent leur temps à rêver de partir en Europe (à lire sur www.fasodansetheatre. com/a-benguer-2006/).
9. Voir, notamment, «50 ans de dépendance", Costard, cravate et pourriture, Abazon, 2010, www. youtube.com/ watch? $v=k h k-n T E e H y o$.

10. Lors de la cérémonie des Koras, victoires de la musique sur le continent africain. Voir l'extrait vidéo : www.youtube.com/ watch?v=ObqEW-Lk8Hw. 11. Voir la page Facebook du Balai citoyen : www.facebook.com/ CitoyenBalayeur?fref $=\mathrm{ts}$.

12. Entretien effectué avec Smockey, octobre 2013, studio Abazon, Ouagadougou. 
opportunités nombreuses, entre musiciens, comédiens, metteurs en scène, scénographes, producteurs et promoteurs, de tous horizons géographiques.

Dans ce contexte de mise en réseaux et de circulations entre différents mondes de l'art (Cefaï, 2015), Smockey est invité à participer à une création de Serge Aimé Coulibaly, chorégraphe burkinabé de danse africaine contemporaine $^{8}$. Né dans les années 1970 à Ouagadougou d'un père burkinabé et d'une mère française, Smockey - contraction de «se moquer »-, part en France au début des années 1990, notamment pour se former aux métiers du son, et revient en 2000 s’installer à Ouagadougou. Il y fonde le studio Abazon («Allez vite » en bissa, sa langue paternelle) devenu aujourd'hui une référence en Afrique de l'Ouest, ce qui lui assure une stabilité financière.

Bien que sa personnalité artistique s'inspire d'influences multiples, et que ses albums soient volontairement éclectiques (musiques traditionnelles, reggae, différents types de rap, slam, chanson humoristique, musique festive, etc.), la reconnaissance de Smockey s'est construite autour d'une image subversive, due à des propos tenus sur le fonctionnement des systèmes politiques africains dans certains de ses textes ${ }^{9}$, ou lors d'apparitions publiques. Engagé notamment dans un combat mémoriel et contre l'impunité (affaire Sankara, puis Norbert Zongo), il est perçu comme une figure médiatique, celui qui ose dire ce que tout le monde pense tout bas, par son public, les acteurs culturels et plus largement de nombreux Burkinabé. Lorsqu'il dédie son trophée de meilleur rappeur africain en 2010 « à l'heure du bilan des indépendances, à tous ceux qui se sont battus, à tous ces grands combattants, Lumumba, Thomas Sankara », devant Blaise Compaoré ${ }^{10}$, son discours est largement commenté localement, vécu par certains comme un véritable acte de bravoure, et par d'autres comme une provocation, irrespectueuse et dérangeante.

Sa réputation se renforce lorsqu'il crée en juin 2013 avec Samsk le Jah, chanteur reggae et animateur radio à Ouagadougou, le Balai citoyen, directement inspiré du mouvement «Y en a marre » au Sénégal (Awenengo-Dalberto, 2011 ; Dimé, 2007). Mouvement politique national sans volonté de prise de pouvoir, le Balai citoyen se définit comme une « sentinelle de la bonne gouvernance et de la démocratie $^{11} \gg$. En s'éloignant des mondes artistiques - le Balai citoyen compte peu d'artistes ou d'acteurs culturels parmi ses membres -, Smockey élargit son réseau et utilise sa notoriété pour rassembler : «Nous, en tant qu'artistes, on a réussi à leur faire comprendre que, nous, on est des outils, des instruments, parce qu'il est évident qu'un artiste aura plus de facilités à attirer l'attention des uns et des autres qu'un citoyen lambda même bardé de diplômes, on s'en fout. Ce n'est pas une figure qu'on reconnaît dans la rue. Donc on a intérêt à faire comprendre que les artistes restent les figures de proue du mouvement parce que c'est nous qui avons attiré cette jeunesse-là et qui vont intéresser cette jeunesse-là ${ }^{12}$. »

Utiliser la reconnaissance et la médiatisation d'artistes engagés auprès de la population burkinabé constitue une des stratégies du Balai citoyen. Inversement, sa rapidité à s'imposer sur la scène politique nationale permet à Smockey d'élargir cette reconnaissance, de créer de nouvelles occasions de 
diffuser sa musique et de se produire sur scène. En effet, de nombreuses manifestations du mouvement s'accompagnent de prestations de Smockey, Samsk le Jah et d'autres artistes sympathisants ou membres du mouvement. Le 16 mai 2015, Smockey et Samsk ont organisé un concert à Bobo-Dioulasso, deuxième ville du pays, en fixant comme condition d'entrée la présentation de la carte d'électeur, dans le cadre d'une campagne de sensibilisation intitulée « Après ta révolte, ton $\operatorname{vote}^{13} »$.

La création Nuit blanche à Ouagadougou, qui imagine et met en scène une nuit insurrectionnelle, est jouée au festival Les Récréatrales ${ }^{14}$ à la fin du mois d'octobre 2014, soit au même moment que les événements insurrectionnels (Bonnecase, 2015). Le temps du festival, le quartier Gounghin se transforme en une «bulle» où différents mondes de l'art se côtoient. Smockey jongle alors entre répétitions, balances et représentations, et les manifestations grandissantes contre la modification de la Constitution, voulue par le parti de Compaoré dans l'objectif de pouvoir briguer un troisième mandat. Il conte cette histoire imaginée sur scène tout en la vivant parallèlement dans les rues de Ouagadougou.

$\mathrm{Si}$ « la ville est, en effet, l'arène où s'affrontent de manière dynamique divers intérêts économiques, sociaux, politiques et parfois culturels » (Diouf, Fredericks, 2013), les circulations de Smockey traduisent différentes temporalités d'une expression politique : d'un côté, des actions du Balai citoyen cherchant à atteindre des objectifs bien précis, ici le départ du président Blaise Compaoré ; de l'autre, des créations artistiques imaginant des soulèvements populaires inspirés de contextes politiques contemporains, avec pour objectif une volonté de changement sociétal à travers une évolution des mentalités, inscrit dans une plus longue durée. Ces formes d'engagement en situation sont légitimées avant tout grâce à l'image engagée du rappeur construite dans une carrière musicale toujours « en train de se faire » (Agier, Ricard, 1997).

Présentée comme «pièce prémonitoire » de l'insurrection burkinabé, Nuit blanche à Ouagadougou est programmée dans plusieurs Instituts français d'Afrique ${ }^{15}$ et en Europe. Parallèlement, le départ de Blaise Compaoré a largement contribué à la médiatisation internationale du Balai citoyen, tout en l'exposant à de nombreuses suspicions ${ }^{16}$. S'imposant comme leader, Smockey apparaît ainsi dans de nombreux reportages, sur Internet, sur France 24 et RFI, notamment ${ }^{17}$. À travers toutes ses activités, il élargit sa visibilité et, donc,

13. Voir à ce sujet: www.droitlibre. net/le-balai-citoyen-une-carted-electeur-pour.html.

14. Festival international de théâtre dans lequel a eu lieu un colloque international « Arts et territoires. Les espaces de la création africaine contemporaine en question ", organisé par Les Récréâtrales, en collaboration avec le Centre des études des médias, des technologies et de l'internationalisation (CEMTI) de l'université de Paris-8, l'université de Ouagadougou, la Coalition des artistes et des intellectuels du Burkina Faso et l'Institut Free Afrik, pour lequel l'auteure a été invitée à présenter une communication.

15. Certains pays ont cependant refusé de la programmer, pour cause d'un possible trouble à l'ordre public et d'une incitation à la révolte. 16. Voir notamment Fodé Diagne (2015).

17. Voir aussi Commeillas (2015) ou Agar (2014).

18. Smockey, «On passe à l'attaque », Pré'volution, Abazon, 2015. 
sa reconnaissance. Dans Nuit blanche à Ouagadougou, Smockey clame un texte présent également dans son cinquième album sorti en mars 2015 :

C'est un peuple qui craque en une nuit blanche

Une danse de tracts que personne ne flanche

Un peuple qui craque en une nuit franche

Une danse d'attaque [...]

On passe à l'attaque d'accord et c'est tout le pays qui s'affole

On ferme les écoles, on sort les pancartes et les banderoles

Partout dans la ville, il y a dans l'air de l'excitation

La place de la nation deviendra place de la révolution $[\ldots]^{18}$.

L’intégration de rappeurs dans différents milieux élargit leur réseau et, par extension, leur reconnaissance, et s'observe à la fois dans des milieux militants et des mondes culturels. Si la danse contemporaine africaine connaît un développement important depuis les années 2000 (Desprès, 2012 ; Andrieu, 2009), le monde du théâtre burkinabé, moins médiatisé, s’institutionnalise et semble de plus en plus connecté à des réseaux internationaux : le festival Les

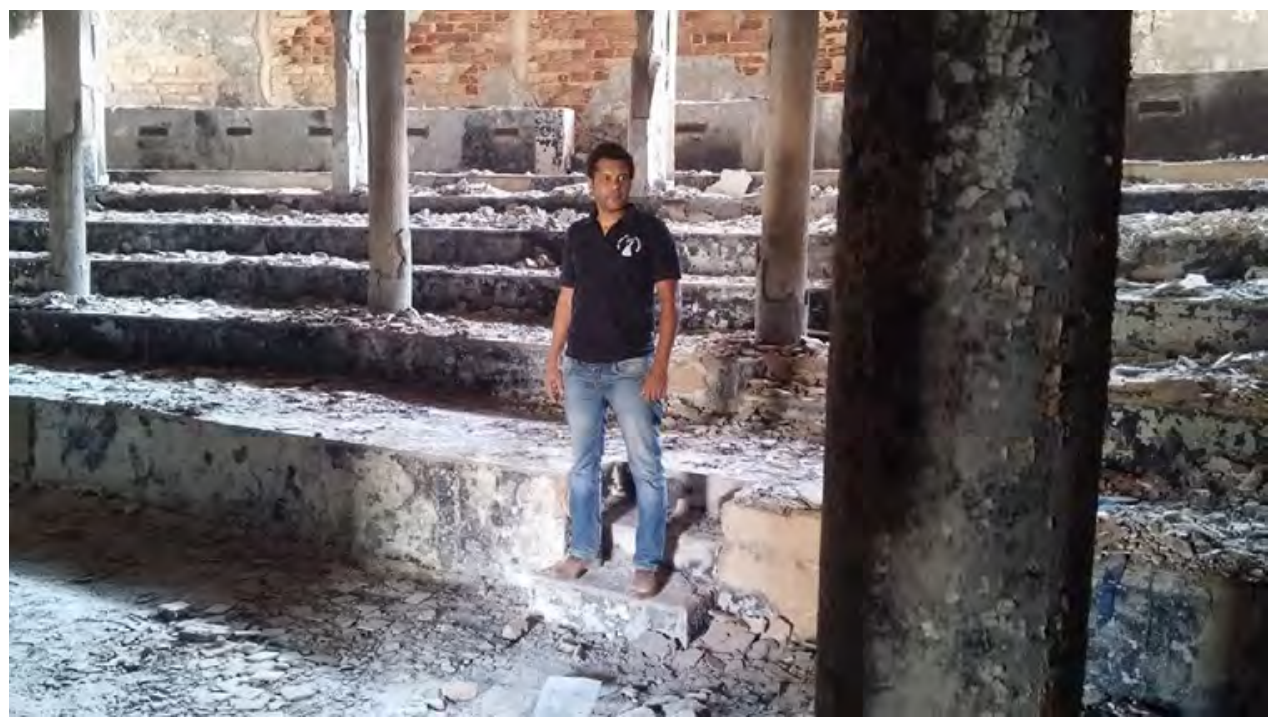

Pré'volution, faire table rase du passé. L'album Prévolution a été présenté à la presse et au public le jeudi 5 mars 2015 devant les bâtiments en ruine de l'Assemblée nationale à Ouagadougou en marge de la campagne «Après ta révolte, ton vote ». Une séance de photos s'est poursuivie à l'intérieur du bâtiment carbonisé. Pré'volution est le titre éponyme du cinquième album de Smockey. Cet album se compose de trois CD symbolisant selon l'artiste, le drapeau burkinabé. Le CD 1 (de couleur jaune) : Prémonition; Le CD 2 (de couleur rouge) : Révolution; et le CD 3 (de couleur verte) : Évolution. La plupart des chansons ont été enregistrées deux ou trois ans avant les évènements insurrectionnels des 30 et 31 octobre 2014. Photo capturée sur le site officiel Facebook de Smockey, 2016. 
Récréatrales grandit chaque année et rassemble des acteurs culturels venus du monde entier.

L'ouverture de ces rappeurs sur d'autres mondes révèle cependant certaines difficultés ancrées dans des rapports de domination. Dans son travail sur les danseurs burkinabé contemporains, la sociologue Altaïr Desprès a par exemple montré que l'apprentissage des codes de la danse contemporaine s'accompagne d'une formation à intellectualiser sa pratique artistique (Desprès, 2014). Autrement dit, un danseur doit savoir expliquer ses choix artistiques en français, langue couramment parlée, et écrite, uniquement par ceux qui ont été scolarisés, ce qui est loin d'être la majorité des artistes d'aujourd'hui. Si dans le monde de la danse contemporaine africaine, les danseurs sont encadrés et formés, les rappeurs burkinabé sont souvent autodidactes. Leurs capacités à s'exprimer en français, à lire et écrire, à s'intégrer à des mondes plus élitistes comme ceux de la danse contemporaine ou du théâtre, dépendent ainsi de leurs parcours et de leurs objectifs de carrière musicale.

Le groupe Faso Kombat, composé de David et Malkhom ${ }^{19}$, fut engagé dans une pièce de théâtre écrite et mise en scène par Aristide Tarnagda, comédien, écrivain et metteur en scène burkinabé. Et si je les tuais tous, madame $?^{20}$ met en scène le monologue d'un exilé, parti à l'aventure en abandonnant ses proches, posté à un feu rouge, qui tente de communiquer avec une automobiliste en lui exprimant ses déceptions, ses angoisses, ses contradictions et sa volonté d'avancer. Le comédien Lamine Diarra est accompagné sur scène par Faso Kombat et un chanteur burkinabé. Aristide Tarnagda évoquait son admiration pour le groupe de rap : «Par rapport à ce que le texte disait, et puis moi je suis un fan dans l'ombre, je les avais déjà vus sur scène en danse avec Serge [Aimé Coulibaly], et je me disais qu'il y avait quelque chose à faire avec eux ${ }^{21}$. »

David et Malkhom découvrent alors le monde du théâtre en France à travers le festival d'Avignon, en juillet $2013^{22}$, où la pièce est programmée pendant quatre jours. En parallèle des représentations, une rencontre entre l'équipe de la pièce et le public est organisée par le festival. Dans ce cadre, David doit faire face à des questions sur les risques de faire du rap en Afrique, dans des contextes jugés violents et verrouillés, à cause des dictatures. Ce jourlà, il répond brièvement que leur musique dérange beaucoup, sous-entendant

19. Créé en 2000 , ce groupe est l'un des plus connus du pays. Chacun évolue désormais en solo.

20. Mise en scène en 2012 , coproduction Les Récréatrales et Institut français.

21. Entretien effectué avec Aristide Tarnagda, Paris, 9 mars 2014. 22. Pour cette dixième édition en 2013, le festival avait souhaité laisser une place importante aux artistes africains, avec le Congolais Dieudonné Niangouna en invité d'honneur, http://www.festivalavignon.com/fr/archives-2013.

23. Le mooré est une des langues nationales les plus parlées dans le pays, notamment dans la capitale.

24. Voir le documentaire sur le rap en Afrique Fangafrika, la voix des sans-voix, Stéphane Rinaldi,

Guillaume Mouillé, Vincent Plassard Laurent Goudet, Pascal Goudet, Renaud Lioult, Staycalm Production, 2007.
25. Faso Kombat, Zem Zem, FK Production, 2013.

26. Dans lequel s'inscrivent des artistes des nouvelles tendances telles que le coupé décalé, les musiques du Nigeria et du Ghana... Ces musiques sont diffusées sur Trace TV. Ces artistes font des tournées internationales organisées par des gros promoteurs et ils reçoivent des cachets importants (un ou plusieurs millions de francs CFA). 
que leurs prises de position explicites constitueraient une menace pour eux, comme pour leurs familles et leurs proches.

Pourtant, le groupe affirmait dans nos échanges informels n'avoir jamais fait l'objet d'intimidations. Cette situation révèle des contraintes ancrées dans des rapports de domination et d'assignation identitaire. David, né à Ouagadougou en 1982, a arrêté l'école en primaire (comme beaucoup d'autres rappeurs), afin de chercher du travail pour subvenir aux besoins de sa famille, après le départ de son père, ancien diplomate, exilé au Ghana depuis le coup d'État de Blaise Compaoré en 1987. Lorsqu'il fonde le groupe Faso Kombat avec Malkhom, il parle à peine le français, il chante et rappe en mooré ${ }^{23}$. Il l'apprend au fur et à mesure, d'abord avec Malkhom, qui rappe en français, mais aussi avec la médiatisation du groupe, nécessitant de donner des interviews, et de faire des tournées en Europe.

Ici, l'intérêt se trouve non pas dans la recherche d'une quelconque vérité, mais bien dans la manière dont certains rappeurs adaptent leur discours à la situation, pour quelles raisons et avec quels enjeux. Sa réaction au festival d'Avignon constitue une forme d'évitement avec un objectif double : en répondant de manière rapide, il contourne les difficultés à argumenter en français. Également, il choisit de donner une réponse attendue, construite sur des représentations occidentales sur la pratique du rap en Afrique $^{24}$, lui permettant d'asseoir une certaine légitimité aux yeux du public présent.

L’intégration de ces rappeurs au sein d'autres milieux constitue cependant une véritable opportunité financière : la pièce est programmée dans une dizaine d'instituts français en Afrique, mais aussi en Europe, et s'accompagne d'une rémunération bien plus importante que les cachets reçus par le groupe pour leurs concerts. De plus, certains séjours ont été l'occasion de proposer une performance musicale de Faso Kombat, également rémunérée, en marge de la pièce. Enfin, les tournées effectuées par le groupe à l'étranger sont par la suite valorisées localement.

\section{S'affirmer contre toute forme d'assignation identitaire}

Aujourd'hui, certains rappeurs burkinabé cherchent à rompre avec cette étiquette d'« artiste engagé ». Loin de la percevoir comme une dépolitisation de ces artistes, cette rupture peut s'appréhender plutôt comme un processus de subjectivation, contre toute forme d'assignation identitaire (Agier, 2013), en réponse à des dispositifs de pouvoir (Mbembé, 2000) ou à des contraintes multiples.

Le travail ethnographique auprès du groupe Faso Kombat a permis de constater un agacement de la part des deux rappeurs face à cette étiquette (Cuomo, 2012). Après avoir sorti leur quatrième album en avril $2013^{25}$, le groupe décide de se séparer suite à des divergences artistiques. David évolue désormais en solo, et sort son premier album en mars 2015. Radicalement différent des créations musicales au sein de Faso Kombat, il se tourne aujourd'hui vers un marché africain plus lucratif ${ }^{26}$, affirmant lors d'un échange informel 
en octobre 2014 à Ouagadougou, être fatigué de jouer la carte de l'« enfant du ghetto engagé » et souhaitant s'ouvrir à d'autres expériences musicales. Toujours inscrit dans le genre «tradi-moderne », David mêle rythmes du «terroir » et sonorités africaines festives en vogue venues de Côte d'Ivoire, du Nigeria et du Ghana principalement.

La reconnaissance - et l'évolution financière - des rappeurs burkinabé se joue majoritairement sur leurs capacités à intégrer des réseaux internationaux, induisant d'effectuer des séjours en France afin de rencontrer des personnalités influentes, démarche suivie par Smarty. D’autre part, rester dans cette catégorie d'« artiste engagé » localement sans soutiens extérieurs l'aurait empêché d'espérer une stabilité financière, d'autant plus que toute forme d'éloignement de certaines réalités socio-économiques du «ghetto » lui aurait été reprochée par son public.

En se tournant vers une esthétique ancrée dans les tendances urbaines africaines, David cherche à construire une carrière musicale spécifiquement africaine, sans avoir à passer par des réseaux internationaux, qui induisent une bonne maîtrise du français, mais aussi des codes culturels permettant d'intégrer certains milieux. Ce genre musical choisi permet ainsi des gains financiers plus rapides, car il s'adresse localement à un autre public, plus large, et se diffuse plus facilement. En outre, des scènes auparavant fermées au groupe, à cause de certains textes jugés trop critiques à l'égard de la politique nationale, lui sont désormais ouvertes ${ }^{27}$. Enfin, il continue de travailler en parallèle sur des créations théâtrales avec Aristide Tarnagda ${ }^{28}$, choisissant d'assumer ses positionnements critiques dans ce milieu plus restreint où engagement politique et stabilité économique ne sont pas incompatibles.

Si certains décident de rompre avec les réseaux internationaux, d'autres cherchent à les intégrer. Smarty évolue également en solo depuis la séparation du groupe Yeleen en 2011. Devenu lauréat du prix Découverte RFI en 2013 avec son premier album, Afrikan Kouleurs ${ }^{29}$, il effectue fréquemment des séjours en France ${ }^{30}$ pendant lesquels l'équipe de RFI s'occupe de lui trouver des interviews dans différents médias, le conseille sur ses choix artistiques et lui présente des producteurs et autres personnalités influentes dans l'industrie musicale française.

Souhaitant d'abord intégrer l'industrie du rap français, il finit par signer avec un producteur parisien spécialisé dans l'industrie musicale française

27. L'auteure pense notamment à des soirées prestigieuses de type gala, rassemblant des élites

financières africaines.

28. Dans une pièce intitulée Façons d'aimer, écrite et mise en scène par Aristide Tarnagda.

29. Smarty, Afrikan Kouleurs, Sawat Production et Umané Culture, 2012.
30. Séjours qui constituent d'importantes données de terrain pour mes recherches.

31. Je reprends une expression employée lors d'un de nos entretiens où il m'expliquait qu'il ne se reconnaissait pas dans cette branche du rap français, trop éloignée de ses propres réalités burkinabé. En revanche, le rappeur Youssoupha, né à Kinshasa et fils du chanteur Tabu Ley Rochereau, constitue l'une de ses sources d'inspiration.

32. Rappeur français reconnu, né en 1977 en Guadeloupe, qui a commencé sa carrière musicale dès son adolescence, au début des années 1990 avec son groupe Ideal J au sein du collectif Mafia Kainfri. II évolue désormais en solo. 
généraliste, déçu par des attitudes qu'il juge trop « ghettos ${ }^{31}$ ». Il connaît également des difficultés à bénéficier d'articles dans la presse spécialisée. Selon Juliette Fievet, membre du jury du prix RFI, manager du rappeur français Kery James $^{32}$, chroniqueuse radio notamment pour l'émission Couleurs tropicales produite par Claudy Siar sur RFI, et chroniqueuse de télévision pour la chaîne France Ô, ces difficultés seraient directement liées à une forme de condescendance : «C'est bien qu'il ait l'image RFI derrière et ça lui permet d'avoir des médias un peu élitistes type Le Monde, Libé, etc., mais ce n'est pas ça qui fait vendre parce que ces médias ne sont pas populaires. Smarty pour moi devrait avoir toutes ses chances évidemment, sauf qu'en fait quand il est venu là récemment faire de la promo, on a essayé de le pousser auprès des médias hip-hop français. Et là, j’ai dû m'embrouiller avec la moitié de mes interlocuteurs, qui m'appellent en pleurant tous les jours pour avoir des infos sur Kery James, qui me parlent de diaspora africaine, nous, on est des renoi [noirs], etc. Et quand je leur parle d'un Smarty, finalement, on m'esquive, c'est même pire qu'esquiver : c'est la condescendance que les Noirs français ont envers les Africains, c'est-à-dire qu'ils sont pires que les Blancs français, tu vois ? C'est-à-dire qu'à un moment donné, on est dans le rap, vous parlez de l'Afrique, vous parlez de vos racines africaines matin, midi et soir, vous bassinez la terre entière avec ce genre de trucs. Et quand t'as un rappeur africain qui, juste soit dit en passant, remplit des stades, dans son pays, voire sur le continent, quand ce mec-là arrive

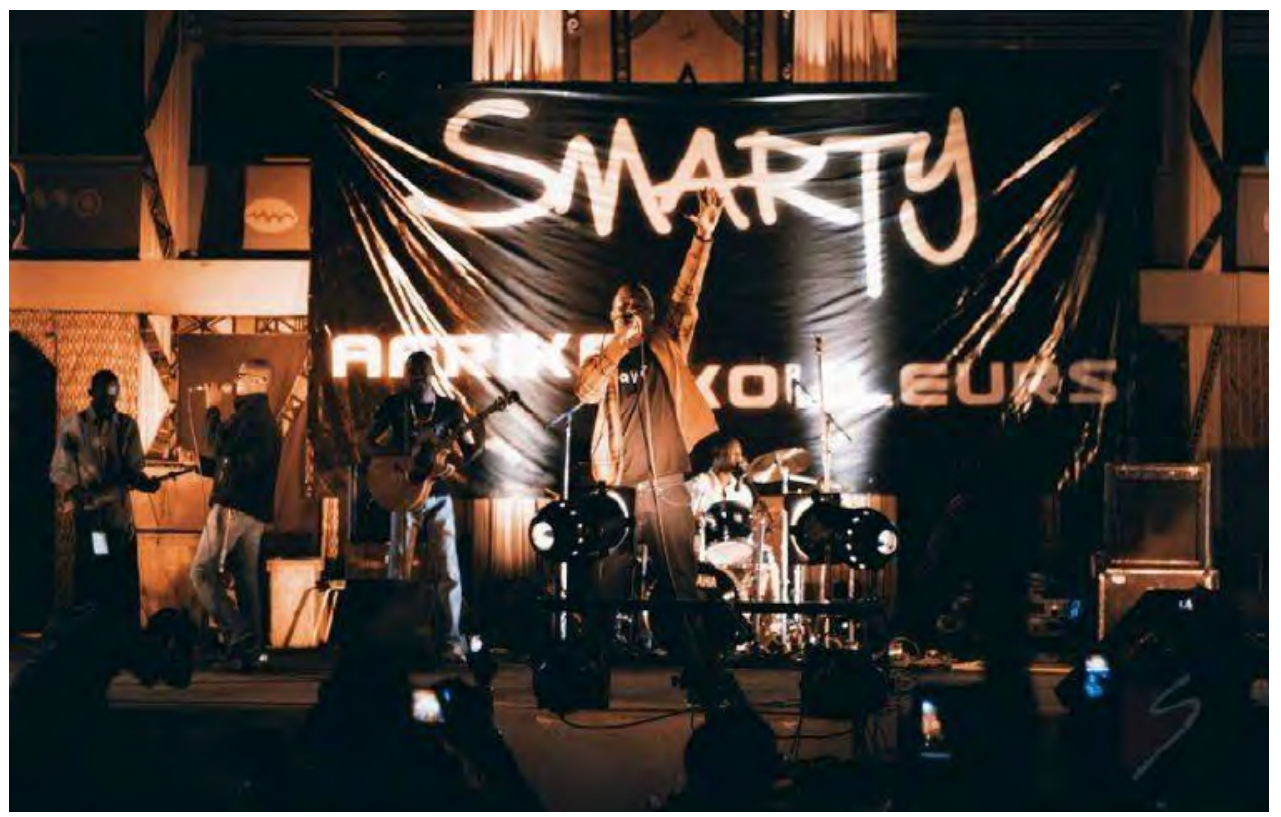

Afrikan Kouleurs. Depuis la séparation du groupe Yeleen en 2011, Smarty évolue en solo. Devenu lauréat du prix Découverte RFI en 2013 avec son premier album, Afrikan Kouleurs, il effectue fréquemment des séjours en France. Photo de Sawat Production, 2013. 
et qu'on vous le propose en interview, ces mecs-là ont une condescendance qui est juste inadmissible, en fait ${ }^{33}$. »

Juliette Fievet considère qu'en tant que rappeur africain, les portes de RFI, sous-entendu de la World Music, lui sont plus facilement ouvertes que celles de l'industrie du rap français, pour les raisons évoquées ci-dessus. Femme influente dans l'industrie musicale parisienne ${ }^{34}$, elle et son équipe cherchent cependant à l'aider afin de toucher des professionnels autres que ceux passionnés d'« Afrique ». Estimant qu'une signature de contrat avec RFI l'enfermerait dans cette case World Music, Smarty finit par signer avec un producteur de variété française. Au-delà d'une question de talent ou de critères artistiques, il maîtrise le français, langue dans laquelle il rappe, mais il est aussi très connecté aux réseaux sociaux, à l'actualité ; il lit et s'inspire beaucoup de romans africains

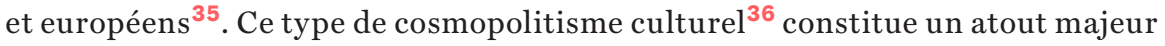
dans ses rencontres professionnelles en France, et détermine la confiance que peut lui attribuer un producteur. Ces mêmes critères étaient soulevés par Béla Bowé, responsable du prix Découverte RFI : « C'est assez subjectif. Cependant, Smarty a été élu à une large majorité donc je pense que chez ce jeune homme on a senti une qualité de texte, de texte d'écriture, de diction, le fait de tenir compte de ses racines africaines, hein, parce que beaucoup de rappeurs, y a de très bons rappeurs en Afrique, le hip-hop représente j'pense plus de $50 \%$, pas loin de $60 \%$ de la production en Afrique. Donc, c'est énorme, mais beaucoup copient soit les rappeurs français, soit les rappeurs anglo-saxons, avec beaucoup moins de moyens, donc le résultat est souvent assez décevant. Mais, lui, il avait une démarche qui est originale qu'ont eue peu d'artistes jusqu'à maintenant. Après, pour l'avoir rencontré, c'est quelqu'un qui parle bien, qui est à l'aise devant un micro, devant une caméra, qui a vraiment une intelligence d'esprit, c'est quelqu'un qui est très futé, et puis en même temps qui a beaucoup d'humilité ${ }^{37}$. »

En signant avec ce producteur, Smarty rompt avec l'étiquette du « rap africain » qui ne pourrait s'exporter qu'à travers les réseaux internationaux militants ou de la World Music. Cette démarche induit des adaptations : pour s'adapter au marché de la variété française, les références africaines doivent être plus globales pour être comprises par le « grand public ${ }^{38}$ » français. Dans son premier album, Afrikan Kouleurs, Smarty utilisait de nombreuses références africaines : Amadou Hampâté Bâ, Kunta Kinte, Samory Touré apparaissent

33. Entretien effectué avec Juliette Fievet, Paris, 12 mars 2014.

34. Évidemment, le monde du rap français est vaste, voir Hammou (2014). Malgré tout, l'industrie du rap à travers la radio privée Skyrock et les majors constitue un réseau d'interconnaissance important.

35. Très peu de rappeurs burkinabé rencontrés aiment lire ou s'inspirent de romans.
36. Je parle ici d'une forme de cosmopolitisme élitiste, "branché ». II faut cependant noter qu'il existe d'autres formes de cosmopolitisme, ordinaire, populaire : sur la musique, voir Stokes (2007).

37. Entretien effectué avec Béla

Bowé, siège de RFI, Issy-lesMoulineaux, 4 mars 2014.

38. L'auteure distingue le grand public d'un public amateur de rap.
Aujourd'hui, Smarty vise un public plus généraliste.

39. Smarty, "Au royaume de mes espérances ", Afrikan Kouleurs, Sawat Production et Umané Culture, 2012, voir le clip vidéo à cette adresse : www.youtube.com/ watch?v=7o-aQUKYPKo. 
dans le morceau «Au royaume de mes espérances ${ }^{39}$ ». Dans l'album en préparation, la chanson «Est-ce que tu réalises » change de ton; l'artiste n'utilise pas le même registre discursif. Au lieu de valoriser une originalité africaine, il prône la ressemblance.

On a les mêmes déprimes, parfois les mêmes blessures,

Mais la même vision prime, en la foi au futur,

Jeunes banlieusards des cités de Brooklyn,

Devenus une icône des disques de vinyles.

$[\ldots]$

Au fond on se ressemble,

En vrai on est pareil,

Que des petits ensembles,

De la même corbeille.

Si des fois il te semble,

Que ta chance sommeille,

La force qui nous rassemble,

Est la clé de toute merveille.

Smarty cherche à s'affirmer au sein d'une élite financière, en contournant toute assignation identitaire figée : il accepte de jouer le rôle de l'artiste africain cherchant à percer en France, tout en diffusant gratuitement sur Internet des morceaux tels que «Bouche B ». Ce texte s'adresse aux Burkinabé à qui il conseille de se méfier des opportunistes de la transition politique en cours au Burkina Faso. La chanson a été largement diffusée en radio à Ouagadougou ; elle lui permet de rester connecté et écouté localement, mais aussi de faire valoir plusieurs facettes de sa personnalité artistique.

Ils étaient « un » la veille dans l'écho,

Firent bouger les masses comme face à Sese Seko.

Jeunesse aux poings endurcis par les mots,

Entends-tu le chaos dans la savane de Yamoussokro?

Mais le fou voit le bouclier se percer,

Depuis que les loups marchent en rang dispersé.

À trop fêter la joie de s'être vengé,

L'on oublie très vite qu'un berger reste un berger. 
Qu'est-ce qui change si face au bâton,

Les fesses du mouton ont gardé la même position,

Hum, plus rien ne sera comme avant,

En un an, on veut que le CNT [Conseil national de la transition]

Fasse plus que 27 ans $^{40}$.

Les cas de Smarty, David et Smockey permettent de saisir les différents contournements stratégiques mis en place par des artistes africains contemporains au sein de leur carrière musicale, laissant entrevoir des appartenances sociales multiples allant d'une certaine élite économique et culturelle à des milieux plus populaires. La reconnaissance internationale en tant qu'artiste engagé acquise d'abord au sein d'un groupe, Faso Kombat pour David, Yeleen pour Smarty, n'engendre pas toujours une volonté de poursuivre une carrière solo dans les mêmes objectifs. Ces ruptures correspondent à une volonté de s'extraire d'un milieu dans lequel on a été catégorisé, et une ambition d'avancer vers une reconnaissance plus large, dans un milieu différent, voire dans un pays différent, en multipliant les circulations et expériences au sein de différents mondes, culturels ou militants, locaux comme globaux.

Comment un artiste engagé pourrait-il se considérer comme tel si d'autres finissent par lui imposer cet engagement? La présente contribution a souhaité montrer l'articulation entre une catégorie restrictive et l'affirmation d'un sujet : isolé des contextes et situations dans lesquels il émerge et évolue, l'engagement artistique en soi ne peut être défini. Il existe toujours en relation avec des contraintes et des rapports de pouvoir changeants. Finalement, la subjectivation se trouve plus dans le contournement d'une catégorie fixe que dans l'affirmation d'appartenir à cette catégorie. Suivre l'évolution de ces artistes constitue une véritable porte d'entrée à l'étude de la relation entre musique, politique et expériences circulatoires. Ici, l'intérêt pour les ruptures s'ancre dans une approche non linéaire de l'objet d'étude qui amène à mieux comprendre la complexité des enjeux et expériences vécues par ces artistes, perçues comme autant de nouvelles « écritures africaines de soi » (Mbembé, 2000).

40. Smarty, "Bouche B », 2015,

www.youtube.com/

watch? $v=$ LdvddwOIVGY. 


\section{Bibliographie}

Agar, L. (2014), « Ouagadougou: à la scène comme dans la rue ». Libération, 11 novembre.

Agier, M. (2012), «Penser le sujet, observer la frontière. Le décentrement de l'anthropologie ". L'Homme, no 203-204, p. 51-75.

Agier, M. (2013), La Condition cosmopolite. L'anthropologie à l'épreuve du piège identitaire, Paris, La Découverte.

Agier, M., Ricard, A. (1997), "Les arts de rue dans les sociétés du Sud », Autrepart, $\mathrm{n}^{\circ} 1$

Amselle, J.-L. (2001), Branchements. Anthropologie de l'universalité des cultures, Paris, Flammarion.

Andrieu, S. (2009), « Le spectacle des traditions. Analyse anthropologique du processus de spectacularisation des danses au Burkina Faso ", thèse d'anthropologie, université Aix-Marseille-1.

Augé, M. (1994), Pour une anthropologie des mondes contemporains, Paris, Flammarion.

Awenengo-Dalberto, S. (2011), "Sénégal : les nouvelles formes de mobilisation de la jeunesse ", Les Carnets du CAP.

Banégas, R., Warnier, J.-P. (2001) "Nouvelles figures de la réussite et du pouvoir ", Politique africaine, $n^{\circ} 82$

Becker, H. (1988), Les Mondes de l'art, Paris, Flammarion.

Bonnecase, V. (2015), « Sur la chute de Blaise Compaoré. Autorité et colère dans les derniers jours d'un régime ", Politique africaine, $n^{\circ} 137$.

Butler, J. (2006), Défaire le genre, Paris, Amsterdam.
Cefaï, D. (2015), « Mondes sociaux. Enquête sur un héritage de l'écologie humaine à Chicago ", SociologieS, numéro spécial « Pragmatisme et sciences sociales ».

Commeillas, D. (2015), « Coup de Balai citoyen au Burkina Faso », Le Monde Diplomatique, avril.

Cuomo, A. (2012), «Entre représentations et stratégies personnelles. Une ethnographie auprès de rappeurs à Ouagadougou (Burkina Faso) ", mémoire de master 2 d'anthropologie, Paris, EHESS, juin.

Cuomo, A. (2014), " Rap et blackness au Burkina Faso. Les enjeux autour de l'accès à une reconnaissance artistique», Politique africaine, $\mathrm{n}^{\circ} 136$.

Daboué, J.B.Y. (2014), Langues nationales et musique moderne burkinabé face à la mondialisation, Paris, L'Harmattan.

Despres, A. (2012), "Se faire contemporain. Les danseurs africains à l'épreuve de la mondialisation culturelle», thèse de sociologie et anthropologie, université Paris-7/ Diderot.

Despres, A. (2014), « Les figures imposées de la mondialisation culturelle. À propos de la socialisation des danseurs contemporains en Afrique ", Sociétés contemporaines, $n^{\circ} 95$.

Dimé, M. (2007), «De bul faale à $Y$ en a marre. Continuités et dissonances dans les dynamiques de contestation sociopolitique et d'affirmation citoyenne chez les jeunes au Sénégal », Codesria.

Diouf, M., Fredericks, R. (2013), Les Arts de la citoyenneté au Sénégal. Espaces contestés et civilités urbaines, Paris, Karthala.
Fodé Diagne, R. (2015), «Y en a marre et Balai citoyen : Ies nouveaux "tirailleurs" de l'impérialisme en Afrique! », Le Journal de l'Afrique, $n^{\circ} 10$, mai.

Frere, M.S., Balima, S.T. (2004), Médias et communications sociales au Burkina Faso. Approche socioéconomique de la circulation de l'information, Paris, L'Harmattan.

Gilroy, P. (2003), L'Atlantique noir. Modernité et double conscience, Paris, Kargo.

Hammou, K. (2014), Une histoire du rap en France, Paris, La Découverte.

Hilgers, M., Mazzocchetti, J.

(2010), Révoltes et oppositions dans un régime semi-autoritaire : le cas du Burkina Faso, Paris, Karthala.

Laplantine, F. (2007), Le Sujet. Essai d'anthropologie politique. Paris, Tétraèdre.

Le Menestrel, S. (2012), Des vies en musique. Parcours d'artistes, mobilités, transformations, Paris, Hermann.

Martin, D.C. (2014), «Attention, une musique peut en cacher une autre ", Volume!, vol. X, $n^{\circ} 2$.

Mbembé, A. (2000a), «À propos des écritures africaines de soi », Politique africaine, $n^{\circ} 77,2000$

Mbembé, A. (2000b), De la postcolonie. Essai sur l'imagination politique dans l'Afrique contemporaine, Paris, Karthala.

Stokes, M. (2007), "On Musical Cosmopolitanism", The Macalester International Roundtable, Institute for Global Citizenship. 